\title{
Spontaneous imbibition in nanopores of different roughness and wettability
}

\author{
Mikhail R. Stukan, ${ }^{* \dagger}$ Patrice Ligneul, ${ }^{\dagger}$ John P. Crawshaw, ${ }^{\ddagger}, \mathbb{9}$ and Edo S. Boek ${ }^{\ddagger}$ \\ Schlumberger Dhahran Carbonate Research Center, Dhahran Techno Valley - KFUPM, \\ P.O. Box 39011, Dammam/Doha Camp 31942, Saudi Arabia, and Schlumberger \\ Cambridge Research, High Cross, Madingley Road, Cambridge CB3 OEL, United Kingdom \\ E-mail: mstukan@slb.com
}

\begin{abstract}
The spontaneous imbibition of liquid in nanopores of different roughness is investigated using coarse grain Molecular Dynamics (MD) simulation. The numerical model is presented and the simplifying assumptions are discussed in detail. The molecular-kinetic theory introduced by Blake is used to describe the effect of dynamic contact angle on fluid imbibition. The capillary roughness is modeled using a random distribution of coarse grained particles forming the wall. The Lucas-Washburn equation is used as a reference for analyzing the imbibition curves obtained by simulation. Due to the statistical nature of MD processing, a comprehensive approach was made to average and smooth the data to accurately define a contact angle. The results are discussed in terms of effective hydrodynamic and static capillary radii and their difference as a function of roughness and wettability.
\end{abstract}

*To whom correspondence should be addressed

†Schlumberger Dhahran Carbonate Research Center, Dhahran Techno Valley - KFUPM, P.O. Box 39011, Dammam/Doha Camp 31942, Saudi Arabia

†Schlumberger Cambridge Research, High Cross, Madingley Road, Cambridge CB3 0EL, United Kingdom

"Current address: Department of Chemical Engineering, Qatar Carbonates and Carbon Storage Research Centre (QCCSRC) Complex Fluids in Porous Media, Imperial College London, South Kensington Campus, London SW7 2AZ United Kingdom

\section{Introduction}

The wetting of solid surfaces controls many processes in both industry and nature. The frame of this work is a better understanding of the mechanisms related to the migration of a fluid in porous media, such as oil in reservoir rocks. If the porous media is exposed to a fluid with an effective contact angle smaller than $\pi / 2$ the fluid will spontaneously imbibe into the pores. When the matrix pores and pore throats are small the capillary forces dominate. In general pore surfaces are rough enough to induce capillary forces different from those expected from studies carried out on non porous flat surfaces. ${ }^{1-3}$ In this study we investigate how roughness influences the rate of imbibition in porous media. ${ }^{4-6}$ Since the geometrical complexity of a natural pore structure is difficult to address, we consider here the simplified case of a single capillary of diameter $\sim 10 \mathrm{~nm}$ with a roughness of size comparable to the size of the fluid molecules. In our study we consider the case when imbibition is affected but not prevented by roughness (no transient pinning phenomena).

Capillary imbibition results in the motion of the meniscus formed between the liquid and its vapor and attached to the wall by a contact line. The apparent contact angle between the meniscus and the wall measured on rough surfaces depends on the surface roughness ${ }^{1-3}$ and the contact line dynamics. ${ }^{7-9}$ The effect of dy- 
namic contact angle on capillary imbibition remains a subject of permanent interest. ${ }^{10-14}$ The fundamental mechanisms involved in the moving contact line dynamics have been recently reviewed by Blake ${ }^{15}$ who compared a number of concurrent models based on hydrodynamics and molecular kinetics. While these models yield to semiempirical and phenomenological description a comprehensive analysis of the process remains to be undertaken.

At the macroscopic scale fluid imbibition in capillaries is described by the Lucas-Washburn equation, which is based on Stokes equations of momentum. Experimental works ${ }^{16-22}$ carried out using nanocapillaries support the validity of the Lucas-Washburn equation used as an ansatz to our study. Though Lucas-Washburn and Stokes equations can be applied down to nanoscales ${ }^{20-26}$ they can not describe behavior of individual molecules, therefore statistical approach naturally applies when considering processes at micro- and nanoscales. ${ }^{7}$ Efficient and widely used tools to study such systems are Molecular Dynamics (MD) ${ }^{24-40}$ and Lattice Boltzmann. ${ }^{41-45}$

Despite the fact that the influence of roughness on a shear flow has been investigated in detail by means of $\mathrm{MD}^{38,46,47}$ and there are analytical results on the influence of regular capillary wall texture, ${ }^{48}$ the impact of random roughness on the imbibition dynamics was not addressed and justifies the present study.

\section{Theory}

It is well known that fluid spontaneously imbibes into a lyophilic capillary. If we assume that: 1) in small channels viscous and capillary forces are dominant, the relaxation of the velocity profile towards a Poiseuille flow (steadystate viscous laminar flow under a fixed pressure drop) is almost instantaneous, thus all inertial effects as well as the effects related to the meniscus formation can be neglected; 2) there is no slip near the capillary wall; 3 ) effects on velocity profile associated with the finite length of the tube and the presence of a meniscus are negligible; 4) the hydraulic resistance of the vapor phase is infinitely small; 5) liquid is incompress- ible; and 6) the contact angle is constant during the motion. Then the rate of liquid penetration can be obtained from the Poiseuille equation:

$$
\frac{d l}{d t}=\frac{R_{D}^{2}}{8 \eta} \frac{\Delta P}{l},
$$

where $l$ is the distance from the meniscus to the capillary entry, $\eta$ is the dynamic viscosity of the fluid, $\Delta P$ is the pressure drop and $R_{D}$ is an equivalent hydrodynamic radius of the capillary. In case of an open-end tube and no gravity:

$$
\Delta P=\frac{2 \gamma \cos \theta_{e}}{R_{V}},
$$

where $\gamma$ is the fluid surface tension, $\theta_{e}$ is the contact angle at equilibrium and $R_{V}$ is the volumetric(static) radius of the capillary $\left(R_{V} \equiv\right.$ $\sqrt{S / \pi}$, where $S$ is the capillary cross section).

The solution of 1 is classical Lucas-Washburn equation: ${ }^{49-51}$

$$
l^{2}(t)=\frac{R \gamma \cos \theta_{e}}{2 \eta} t+l(0)^{2} .
$$

Here $l(0)$ is the initial position of the meniscus at time $t=0$ and $R=R_{D}^{2} / R_{V} \cdot{ }^{16}$ In an ideally smooth capillary and in the case of no slip boundary condition $R=R_{D}=R_{V}$, for rough capillaries this is not true any more.

3 was obtained with simplifying assumptions. Since the saturated pressure of typical hydrocarbons at moderate temperature is very small the vapor resistance can be safely neglected compared to the driving capillary pressure gradient (assumption number 4). All the effects associated with a possible slip can be taken into account through $R_{D}$, as shown by Dimitrov et al. ${ }^{36}$ The assumptions about velocity profile relaxation, absence of inertia and gravity, finite size of the capillary and fluid incompressibility are discussed in detail later. In general the dynamic contact angle depends not only on the velocity of the moving contact line, but also on nonlocal hydrodynamic effects. ${ }^{52,53}$ However, in our case [nano scales, low Reynolds and Weber numbers $(R e \sim 0.01, W e \sim 0.001)]$ the variation of contact angle during the motion can be considered as a simple function of the meniscus velocity, $d l / d t$, i.e. $\theta=f(d l / d t)$. For this 
purpose the classical Lucas-Washburn equation (3) is modified by substituting a velocitydependent dynamic contact angle $\theta$ for $\theta_{e}$ in 2 . Among the several expressions proposed in the literature for $f(d l / d t)^{15,54-60}$ we follow the molecular-kinetic theory approach suggested by Blake. ${ }^{61}$

According to this theory the macroscopic behavior of the moving wetting line depends on the overall statistics of individual displacements of molecules in its vicinity. The velocity of the moving wetting line depends on the frequency of molecular displacement, $K_{0}$, and the average length of such displacement, $\lambda$. In the simplest case $\lambda$ is of the order of the distance between adsorption sites on the solid surface. The driving force for the motion of the wetting line is a nonequilibrated surface tension force $\gamma\left(\cos \theta_{e}-\cos \theta\right)$. Eyring's activated-rate theory for transport in liquids provides:

$$
\frac{d l}{d t}=2 K_{0} \lambda \sinh \left[\frac{\gamma \lambda^{2}\left(\cos \theta_{e}-\cos \theta\right)}{2 k_{B} T}\right],
$$

where $k_{B}$ is the Boltzmann's constant, and $T$ is the temperature. For small values of the argument of sinh (when the deviation of the contact angle from its equilibrium value is not too large) 4 can be linearized to:

$$
\frac{d l}{d t}=\frac{K_{0} \lambda^{3}}{k_{B} T}\left[\gamma\left(\cos \theta_{e}-\cos \theta\right)\right],
$$

or

$$
\cos \theta=\cos \theta_{e}-\frac{\xi}{\gamma} \frac{d l}{d t}
$$

where $\xi=k_{B} T /\left(K_{0} \lambda^{3}\right)$ is a coefficient of so called "wetting line friction," which depends on both fluid viscosity and liquid solid interaction. ${ }^{62}$ Since in the beginning of the motion the moving meniscus is almost flat, the linearization works better for weakly wetting surfaces where the value of equilibrium contact angle is close to $\pi / 2$.

Substituting in $2 \cos \theta_{e}$ by its dynamical equivalent (6) one obtains:

$$
l \frac{d l}{d t}=\frac{R}{4 \eta} \gamma\left[\cos \theta_{e}-\frac{\xi}{\gamma} \frac{d l}{d t}\right]
$$

as previously, $R=R_{D}^{2} / R_{V}$. Martic et al. suggested in Ref. 32 an analytical solution of 7 in the following form. 7 can be written as:

$$
1-P_{2} \frac{d l}{d t}-P_{3} l \frac{d l}{d t}=0,
$$

here $P_{2}=\xi /\left(\gamma \cos \theta_{e}\right), P_{3}=4 \eta /\left(R \gamma \cos \theta_{e}\right)$. With the initial condition $l(0)=P_{1}, 8$ has the exact solution

$$
l(t)=-\frac{P_{2}}{P_{3}}+\sqrt{\frac{2 t}{P_{3}}+\left(P_{1}+\frac{P_{2}}{P_{3}}\right)^{2}} .
$$

In case of $P_{2}=09$ tends to the classical LucasWashburn equation. All the effects related to the dynamic contact angle depend on the ratio $P_{2} / P_{3}=R \xi / 4 \eta$. The parameters $P_{1}, P_{2}, P_{3}$ can be obtained from the fitting of the simulation data with 9 .

To calculate $\cos \theta_{e}$ let us rewrite 8 as

$$
\frac{d l}{d t}=\frac{1}{P_{3}\left(l+P_{2} / P_{3}\right)} .
$$

Then, taking into account $\xi=P_{2} \gamma \cos \theta_{e}$ from 6 one obtains

$$
\cos \theta=\cos \theta_{e}\left[1-P_{2}\left(\frac{d l}{d t}\right)\right] .
$$

Eliminating $d l / d t$ from 10 and 11 gives

$$
\cos \theta=\frac{l(t) \cos \theta_{e}}{l(t)+P_{2} / P_{3}} .
$$

This equation can be used to determine $\cos \theta_{e}$ by fitting the simulation data using previously calculated $P_{2}$ and $P_{3}$.

\section{Model}

In this study we considered the processes of spontaneous imbibition of dodecane into horizontal capillaries/pores of approximately $10 \mathrm{~nm}$ in diameter and up to about $100 \mathrm{~nm}$ length. We used the coarse-grained model suggested by Marrink et al. ${ }^{63,64}$ for which one simulation particle represents few methylene $\left(\mathrm{CH}_{2}\right)$ or/and methyl $\left(\mathrm{CH}_{3}\right)$ groups. We assumed that the fluid in the simulation corresponds to do- 
decane (one dodecane molecule is represented by three coarse grained (CG) particles connected by elastic bonds) under normal conditions ( $T=300 \mathrm{~K}$ and $P=1 \mathrm{~atm}$ ).

For the simulation the ESPResSo package ${ }^{65}$ was used. The spatial interaction between $\mathrm{CG}$ particles $i$ and $j$ separated by the distance $r$, is described by a spherically truncated and shifted Lennard-Jones (LJ) potential:

$$
U(r)= \begin{cases}U_{L J}(r)-U_{L J}\left(r_{c}\right), & r<r_{c} \\ 0, & r \geq r_{c}\end{cases}
$$

with

$$
U_{L J}(r)=4 \varepsilon_{i j}\left[\left(\frac{\sigma_{i j}}{r}\right)^{12}-\left(\frac{\sigma_{i j}}{r}\right)^{6}\right] .
$$

The parameter $\sigma_{i j}$, which defines the effective minimum distance between particles $i$ and $j$, was set to 1 for all pair interactions and corresponds to $0.47 \mathrm{~nm}$ in SI units. The cut-off radius is set to $r_{c}=1.2 \mathrm{~nm}=2.553191 \sigma$. The interaction parameter $\varepsilon_{i j}$ depends on the types of particles involved in the interaction. For oil-oil volume interactions $\varepsilon_{o o}=3.4 \mathrm{~kJ} / \mathrm{mol}$ $=1.36309 k_{B} T$. The strength of pair interactions between oil particles and particles forming the capillary walls, $\varepsilon_{o w}$, was a variable in our study. Hereafter, all lengths are quoted in $\sigma$ units and energies in $k_{B} T$ units, if not specified otherwise.

Bond interaction between neighboring beads along the hydrocarbon molecule was modeled by the harmonic potential:

$$
U_{\text {bond }}(r)=\frac{1}{2} K_{\text {bond }}(r-1)^{2},
$$

The chain stiffness was modeled by a cosine square bond angle potential:

$$
U_{\alpha}(\vartheta)=\frac{1}{2} K_{\alpha}(\cos (\vartheta)-1)^{2}
$$

where $\vartheta$ is the angle between two consequent bonds along the chain. The values of constants in 15 and 16 were set to $K_{\text {bond }}=$ $1250 \mathrm{~kJ} / \mathrm{mol} / \mathrm{nm}^{2}$ and $K_{\alpha}=25 \mathrm{~kJ} / \mathrm{mol}$.

All particles are considered to be uncharged. Each dodecane molecule is represented by 3 coarse grained beads, thus the mass of each coarse grained particle is $56.78 \mathrm{amu}$ (considered as a unit mass, $m$, in our simulation).

The temperature of the liquid was kept constant at $\mathrm{T}=300 \mathrm{~K}$ using the standard dissipative particle dynamics (DPD) thermostat ${ }^{66-68}$ with a friction parameter $\zeta$ set to 0.1 and a step-function-like weight function with a cutoff equal to $r_{c}$.

The equations of motion were integrated with the standard velocity Verlet algorithm ${ }^{69}$ with a timestep $\Delta t=0.01 \tau\left(\tau=\sigma \sqrt{m / k_{B} T}\right.$ is the basic time unit).

\section{Fluid properties}

The following values for physical parameters of the fluid were obtained.

Density and compressibility: A simulation in the isothermal-isobaric ensemble (NPT) was performed to calculate the isothermal compressibility $\beta$ and bulk density $\rho_{\text {bulk }}$ of the simulated hydrocarbons. The simulation system contained 10000 hydrocarbon molecules (30000 CG particles). The isothermal compressibility $\beta$ calculated from the volume fluctuations ${ }^{70}$ $\left(\beta=14.2 \cdot 10^{-10} \mathrm{~Pa}^{-1}\right)$ is of the same order of magnitude as the experimental one (9.88 . $\left.10^{-10} \mathrm{~Pa}^{-1}\right)$. The bulk density was found to be $763 \mathrm{~kg} / \mathrm{m}^{3}\left(\rho_{\text {bulk }}=0.842 \sigma^{-3}\right)$ and matches the experimental value $\left(744 \mathrm{~kg} / \mathrm{m}^{3}\right)$ reasonably well.

Surface tension: The surface tension was obtained from the anisotropy of the pressure tensor $P_{\alpha \beta}$ during simulation (in canonical ensemble (NVT)) of a slab of liquid dodecane in thermodynamic balance with the vapor phase:

$$
\gamma=\frac{L_{z}}{2}\left[<P_{z z}>-\frac{1}{2}\left(<P_{x x}>+<P_{y y}>\right)\right]
$$

$z$ corresponds to the direction perpendicular to the slab, and the simulation box has size $L_{x} \times$ $L_{y} \times L_{z}$.

To check the occurrence of any finite size effect, the measurements were performed for two systems. Simulation box of size $25 \sigma \times 25 \sigma \times 40 \sigma$ 
(system contained 9999 CG particles) and simulation box of size $50 \sigma \times 50 \sigma \times 100 \sigma$ (system contained $120000 \mathrm{CG}$ particles). The values obtained are $\gamma_{9999}=23.4 \pm 0.8 \mathrm{mN} / \mathrm{m}$ and $\gamma_{120000}=23.0 \pm 1.4 \mathrm{mN} / \mathrm{m}$. Both results are very similar and are in good correspondence with the experimental data $(\gamma=24.75 \mathrm{mN} / \mathrm{m})$.

To check that the presence of liquid-vapor boundary does not create any undesirable artificial density reduction of the liquid phase, we have checked the density profiles across the simulation box. The transition regions between vapor and fluid phases are very narrow. This observation corresponds well with the fact that saturated vapor pressure of dodecane is very small $(\sim 20 \mathrm{~Pa}$ at $T=300 \mathrm{~K})$.

\section{Time unit}

While such variables as density, length scales, temperature, pressure and energy keep their physical meaning in the coarse grained system, it is not exactly the case for the time scale. The dynamics of processes in coarse grained systems is usually faster in comparison to fully atomistic description due to reduction in degrees of freedom and the elimination of fine interaction details.

One method to match the simulation time and the real time is to analyze diffusion in the system and thus obtain the scale of the time axis by matching simulation and experimental values of the diffusion coefficients.

During the NPT simulation used for calculation of the density and compressibility the mean squared displacement, $\left\langle r_{D}^{2}\right\rangle$, was measured. $\left\langle r_{D}^{2}\right\rangle$ is a linear function of time of slope $0.063 \sigma^{2} / \tau$. Using Einstein's equation for diffusion $\left(\left\langle r_{D}^{2}\right\rangle=6 D t\right)$ and the experimental value of self diffusion coefficient for dodecane ${ }^{71}$ equal to $D_{C_{12} H_{26}}=1.0 \cdot 10^{-9} \mathrm{~m}^{2} / \mathrm{s}$ we obtained the effective value of simulation time unit $\tau \approx 2.32 \mathrm{ps}$.

Direct estimate of the time unit from the MD units gives $\tau=\sqrt{\sigma^{2} m / k_{B} T} \approx 2.24 \mathrm{ps}$.

Thus, one simulation time unit corresponds to $2.2-2.3 p s$ of real time. In order to link the simulation data to the real physical units $\tau=2.3 \mathrm{ps}$ was used. As a result, the simulated fluid viscosity, measured by the shear rate method, ${ }^{72}$ was found to be $\eta=0.972 \pm 0.2 \mathrm{mPa} \cdot \mathrm{s}$, which is of the same order of magnitude as the experimental one $(\eta=1.314 \mathrm{mPa} \cdot \mathrm{s})$.

\section{Capillaries}

In order to build rough capillaries we confined a melt of random AB-copolymer (6-mer with the average $\mathrm{A}: \mathrm{B}$ ratio equal to $4: 1$ ) between two concentric cylinders of radii $12 \sigma$ and $17 \sigma$ and $200 \sigma$ length. The monomer units of type A were allowed to trespass the inner wall, while the monomer units of type B remained constrained between the cylindrical boundaries. After equilibration the melt was frozen resulting in a specific roughness of the capillary. All particles not connected to the capillary wall were then manually removed from the system. Afterwards, to reduce the number of particles in the simulation all the particles for which the distance, $d$, from the capillary central line was more than $15 \sigma$ were eliminated. As a result of these treatments, the "initial" capillary of length $L=200 \sigma$ was created.

Particles in the capillary walls are in nonordered glassy (frozen liquid) state. The wall particles were fixed to keep the roughness constant during the run (the similar approach was used by Gelb and Hopkins ${ }^{24}$ ). In order to check a possible effect of the thermal agitation of the wall particles, a few test simulations were evaluated with both dynamic and static walls. No discernible differences in rising dynamics were noticed, consistent with the observations of other authors. ${ }^{33,40}$

The capillary roughness, $\mathcal{R}$, is the main characteristic we are interested in. The definition of roughness proposed by Wenzel, ${ }^{1}$ according to which $\mathcal{R}=\frac{\text { actual surface }}{\text { geometric surface }}$, is not applicable in our case due to the complex shape of the roughness (random roughness) and discrete structure of the surface (the way to calculate the "actual surface" in such circumstances is not clear). We used the root mean square roughness definition, which is commonly used in surface 


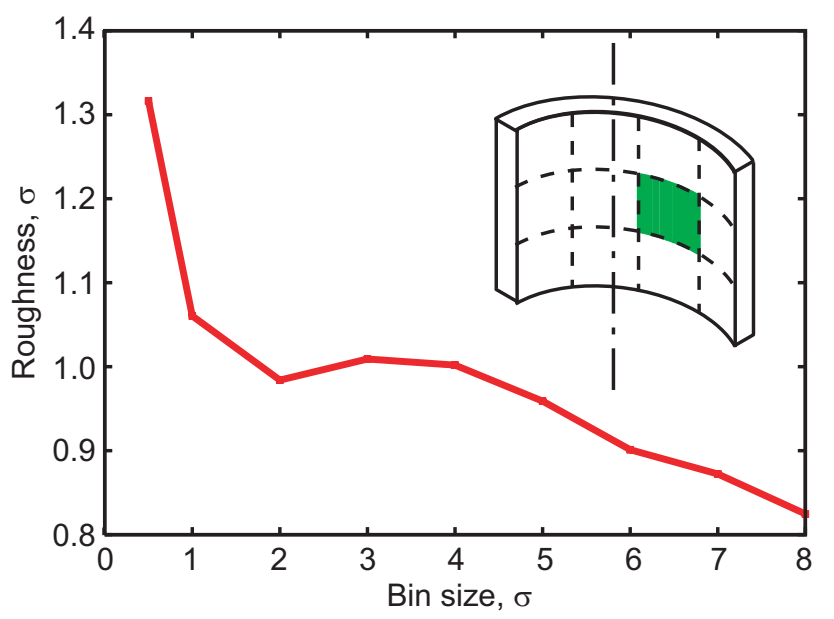

Figure 1: Capillary roughness as a function of the bin size.

science: ${ }^{73}$

$$
\mathcal{R}=\sqrt{\frac{\sum_{i=1}^{N}\left(d_{i}^{s}-\left\langle d_{i}^{s}\right\rangle\right)^{2}}{N-1}} .
$$

Where $d_{i}^{s}$ is the shortest distance from the capillary central line to the wall particles in the $i$-th bin; $\left\langle d_{i}^{s}\right\rangle$ is the average distance, and $N$ is the total number of bins. In the case of a cylindrical capillary the size of the bin is defined by the slice thickness and the arc length (inset in 1 ). The latter depends on the distance from the center.

Although the value of roughness depends on the bin size used for the calculations of $d_{i}^{s}$, in certain interval of bin sizes, $b$, roughness is nearly constant (1) illustrating a fractal behavior of the surface.

We have chosen the bin size to be $4 \sigma \times 4 \sigma$, that corresponds to the plateau region for $\mathcal{R}(b)$ dependence, and also was used in experiments on nanoroughness. ${ }^{74}$ The capillary was divided into 20 angular segments and cut into slices of width equal to $4 \sigma$. In the case of 20 segments, the segment arc length is equal to $4 \sigma$ at $d \approx 12.73 \sigma$. This distance is in the region where the radial density profile of the wall particles approaches the plateau corresponding to the bulk wall density $(\sim 0.9)$ (see inset in 2$)$.

The roughness of the initial capillary was found to be $1.002 \sigma$.
Table 1: Orthographic projections of long $(200 \sigma)$ capillaries of different roughness.

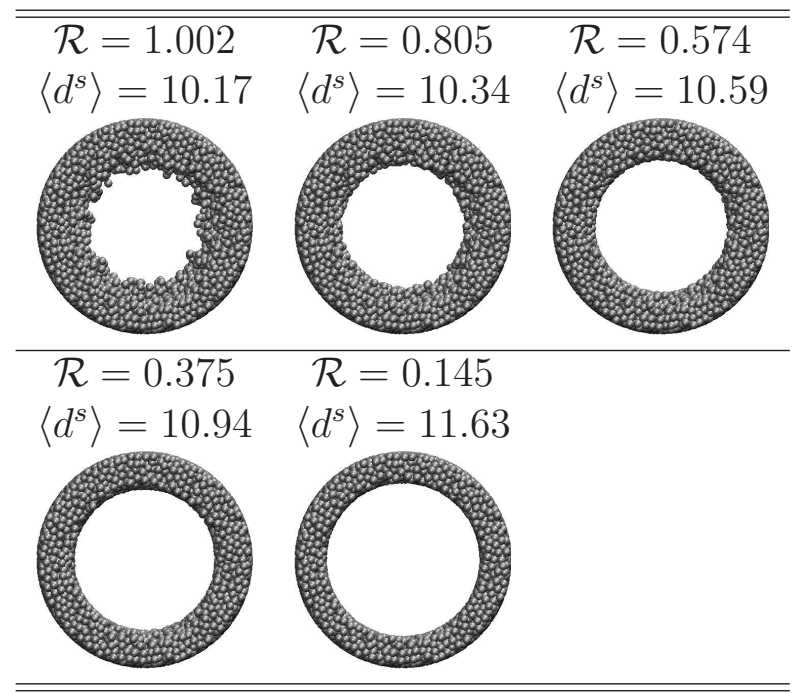

Capillaries of different roughness were obtained by "polishing" the inner surface of the initial capillary. This has been done by eliminating the particles located closer than a defined distance from the central line. With this method smoother capillaries with roughness equal to $0.805,0.574,0.375$ and 0.145 were created covering a range from significantly rough to almost perfectly smooth (see 1 ). These capillaries have length equal to $200 \sigma$ and are referred to as "long".

Since we expect the imbibition data for rough capillaries to be rather noisy, in order to increase the statistics we performed additional simulations using capillaries of half length of the original ones, except for the smoothest one. Reducing the capillary length by two reduces the imbibition time by four, allowing the production of many more statistical samples. Two separate sets of short capillaries were created. The first one was obtained by removing the downstream half of the long capillaries (these capillaries are referred to as "short"). The second set was built using the middle sections of the long capillaries (from $30 \sigma$ to $130 \sigma$ ). These capillaries are referred to as "shifted". This has been done to eliminate possible bias due to particular features of roughness. Roughnesses of short capillaries differ slightly from the long ones: the "short" set roughness values are 0.381, 0.578, 


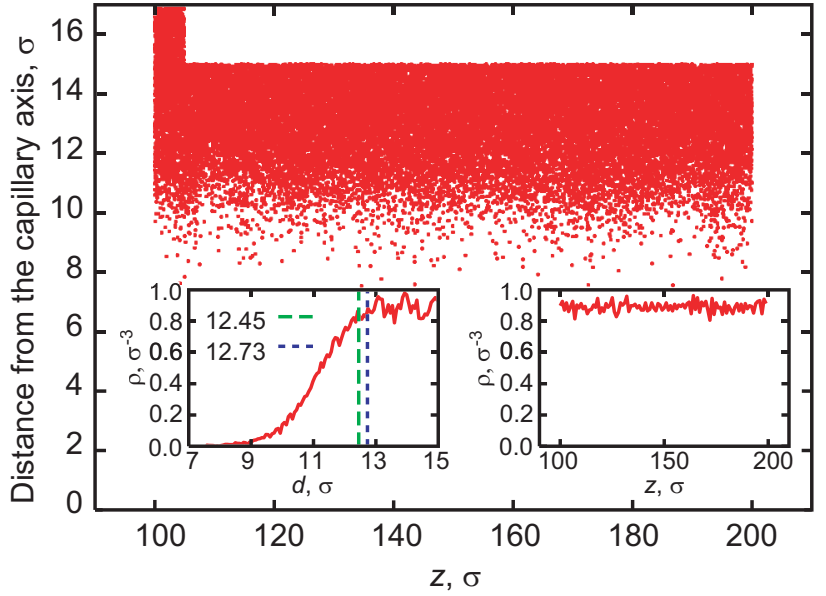

Figure 2: Positions of the wall particles for capillary of $100 \sigma$ length (roughness $\mathcal{R}=1.015$ ). Number density of wall particles across the capillary (left inset) and in the "bulk" wall $(12.5 \sigma<$ $d<15 \sigma)$ (right inset).

0.806 and 1.015, and the "short-shifted" set values are $0.348,0.544,0.771,0.974$.

The role of roughness may be affected by wettability. Therefore we varied the capillary wettability by means of the oil-wall interaction parameter $\varepsilon_{\text {ow }}$ (see 14). This parameter was varied between 1.10 and 1.40. The upper limit 1.40 of oil-wall interactions corresponds to the situation of a complete wetting (for oil-oil pair interactions $\varepsilon_{o o} \approx 1.36$ ), while for $\varepsilon_{o w}=1.10$ the contact angle is close to $\pi / 2$ which marks the region of $\varepsilon_{\text {ow }}$ where spontaneous imbibition can take place. The relation between the interaction parameter $\varepsilon_{\text {ow }}$ and the equilibrium contact angle can be estimated based on the data shown in 3. Hereafter we will omit the subscripts for the oil-wall interaction parameter $\varepsilon_{\text {ow }}$.

The simulations for the long capillaries were performed for $\varepsilon$ equal to $1.10,1.20,1.30$ and 1.40. For the smoothest capillary the simulation was also performed for $\varepsilon=1.35$. For the short capillaries we added cases with $\varepsilon$ equal to $1.15,1.25,1.35$ in order to refine the study of wettability effects.

\section{Simulation System}

The size of the simulation box in all cases is $50 \sigma \times 50 \sigma \times 310 \sigma$. Simulations were performed in NVT ensemble. Periodic boundary condi- tions are applied in the directions perpendicular to the capillary axis. To make the system closer to the model of a rock pore, an additional plane of $5 \sigma$ width perpendicular to the direction of the capillary axis was set up around the capillary entry to simulate the surface in which the pore exists. The plane was created from atoms of the same type as the capillary. The plane and the capillary are placed in the simulation box fixing the capillary entry at $z=100 \sigma$ (see 2). Thus in the beginning of the simulation all the fluid particles are located in the reservoir of size $50 \sigma \times 50 \sigma \times 100 \sigma$. To avoid the accumulation of the fluid particles at the far end of the capil$\operatorname{lary}^{24,36}$ we have set up an open-end capillary, with space in front of the capillary end allowing particles to evaporate. A sample cross section of the simulated system is shown in 3 .

For the long (length 200 $\sigma$ ) capillaries the simulation system contains 40000 dodecane molecules (120000 CG particles) and consists of about $180000 \mathrm{CG}$ particles in total (the exact value differs slightly for capillaries of different roughness, since the number of particles forming the wall is different). For the short (length $100 \sigma$ ) capillaries the number of dodecane molecules is reduced down to 20000 (60000 CG particles). The size of both systems is big enough to create a sufficient volume of bulk fluid so that the fluid in the capillary is not affected by the liquid-vapor boundary in the reservoir.

To make the runs less correlated the initial configurations used for the cases of short and long capillaries were set differently. For long capillaries we used the initial configuration with a liquid already inside the capillary at certain distance from the inlet. This also eliminates the impact of the processes taking place at the very beginning of the imbibition ${ }^{31}$ close to the capillary inlet. This configuration was built by setting an impermeable barrier at $l=5 \sigma$ limiting the further penetration of the fluid inside the capillary. The system has been equilibrated for 20000 timesteps at the given interaction parameter $\varepsilon$ with the barrier, then the barrier was removed and the spontaneous imbibition inside the main part of the capillary began. In the case of short capillaries the initial impermeable 


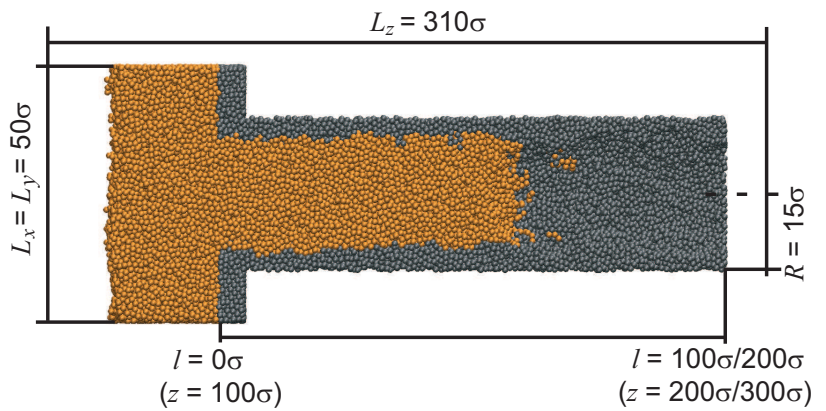

Figure 3: Snapshot of the simulation system.

barrier was located at $l=0 \sigma$, so that the fluid was not able to enter the capillary at all. As for the case of long capillaries, each system was equilibrated for 20000 timesteps at a given interaction parameter $\varepsilon$ with the presence of the barrier, then the barrier was removed and the imbibition process was monitored.

Since in the simulation the wall particles were fixed, we investigated how this fact influences the fluid density profiles ${ }^{75}$ inside the capillary. These profiles are shown in 4. A small layering near the walls is observed for smooth capillaries only $(\mathcal{R}=0.145$ and $\mathcal{R}=0.375)$, the layering disappears as the roughness increases. The density of the fluid inside the capillary is close to its bulk value $\left(0.842 \sigma^{-3}\right)$. The profiles were obtained for particles located between $50 \sigma$ and $150 \sigma$ from the capillary inlet (long capillaries) and averaged over all conformations for which the meniscus level is above $150 \sigma$. The data plotted in 4 are for $\varepsilon=1.40$; for smaller values of oil-wall interaction the correlations are even less pronounced.

We also checked that the fluid particles are not under influence of the external wall of the capillary (they do not come closer than the cutoff distance $r_{c}$ ). As one can see from the inset in 4 the number of particles behind the boundary $d=12.45 \sigma$ is negligible $(\sim 0.03 \%)$.

\section{Validity of the assumptions about the imbibition conditions}

The following assumptions made as a frame for the derivation of the Lucas-Washburn equation (3 and 9) are discussed below: the absence of gravity and the fluid incompressibility, the instantaneous relaxation towards the viscous regime and the finite size of the meniscus.

Absence of gravity and fluid incompressibility: A virtual gravity force acting on an individual $\mathrm{CG}$ particle is $f=m g \approx 9.25$. $10^{-25} \mathrm{~N}$. Typical values of interaction forces in the system are of the order of one in LJ units $\left(1\left[k_{B} T / \sigma\right] \approx 8.81 \cdot 10^{-12}[N]\right)$. It means that the effect of gravity would be noticeable for systems containing $\sim 10^{13}$ particles and can be safely neglected in the current study. The same conclusion is provided by continuum fluid mechanics analysis. ${ }^{76}$

The compressibility results in relative volume variation due to the pressure drop during the imbibition: $\Delta V / V=\beta \Delta P=2 \beta \gamma \cos \theta / R_{V}$. For a strongly wet $(\cos \theta=1)$ capillary of $R_{V} \sim 10 \sigma$, this leads to an extension of the fluid column of about $1 \%$. Therefore when required accuracy is lower than $1 \%$ fluid can be considered as incompressible.

Transient regime: The time for the meniscus formation, $\tau_{0}$, and the duration of the inertial regime which follows the moment of contact between the capillary and the liquid bath (after the meniscus creation), $\tau_{1}$, can be estimated ${ }^{77}$ as $\tau_{0} \sim\left(\rho R^{3} / 8 \gamma\right)^{1 / 2}$ and $\tau_{1} \sim \rho R^{2} / 4 \eta$.

For dodecane in a capillary of $\sim 10 \mathrm{~nm}$ in diameter the maximum value of the time required to reach the viscous regime is $\tau^{*}=\tau_{0}+\tau_{1} \sim$

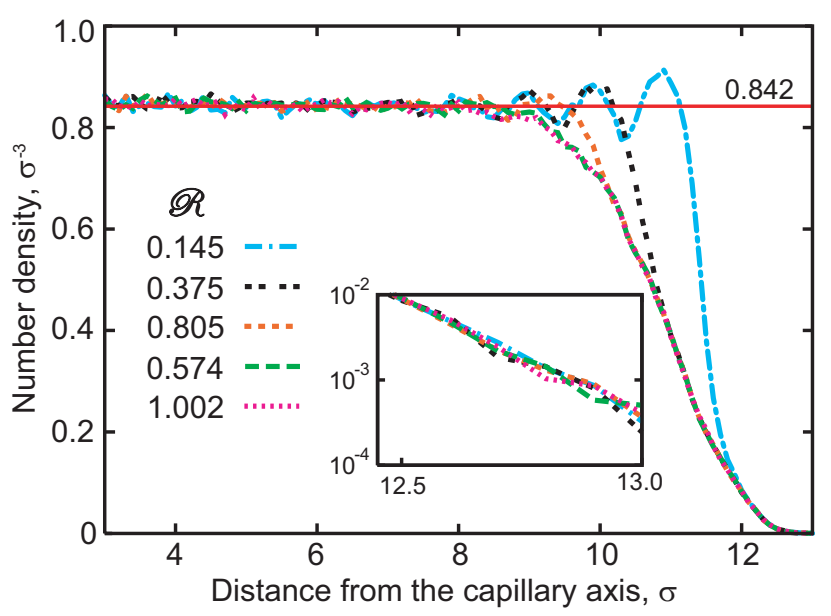

Figure 4: Radial density profile of the fluid inside capillaries of different roughness, $\varepsilon=1.40$. 
30 ps, after which the Lucas-Washburn equation can be applied. It means that in our simulations the transition from inertial to viscous regime takes place almost instantaneously.

Meniscus position and contact angle: In the inset in 5 the fluid density profile is plotted versus the distance along the capillary axis. At the interface between the fluid and its vapor a transition is discernible reflecting the shape of the meniscus. Therefore the exact location and shape of the meniscus require additional analysis. In order to define the shape of the meniscus we have divided the capillary into 20 coaxial cylinders (bins) of equal volume, from the central line up to the radial distance, $R_{\theta}$ where the contact angle is measured. For each bin the density profile along the capillary has been calculated. The location where the fluid density reaches $50 \%$ of its bulk value $\left(\rho_{\text {bulk }} / 2=0.42 \sigma^{-3}\right)$ was considered as the liquid-vapor interface inside each bin. Thus we obtained 20 points representing the meniscus interface in the coordinate frame: distance from the capillary axis, distance from the capillary entry. We assume the meniscus to have a hemispherical shape (see 5), therefore the discrete interface was approximated by a circular arc. A circular arc is shown to be appropriate to fit the meniscus ${ }^{14,25,31,32,37,40}$ apart from a region very close to the wall where, especially in the case of a prewetting layer existence, some deviations can occur. ${ }^{78}$ Although some traces of prewetting film were observed at high values of $\varepsilon$ they are related to individual fluid molecules adsorbed in the roughness maze rather than constituting a continuous prewetting fluid layer. This region was omitted in data fitting.

Table 2: Average values of the residual $\aleph$ (data for long capillaries).

\begin{tabular}{l|cccc}
\hline \hline $\mathcal{R}$ & \multicolumn{4}{|c}{$\varepsilon$} \\
\cline { 2 - 5 } & 1.10 & 1.20 & 1.30 & 1.40 \\
\hline 0.145 & $1.95 \pm 0.87$ & $1.89 \pm 0.81$ & $2.49 \pm 6.67$ & $1.64 \pm 0.69$ \\
0.375 & $3.07 \pm 1.67$ & $3.36 \pm 1.87$ & $3.28 \pm 2.61$ & $2.48 \pm 2.64$ \\
0.574 & $5.21 \pm 5.15$ & $4.67 \pm 3.41$ & $3.88 \pm 3.19$ & $3.45 \pm 3.37$ \\
0.805 & $5.68 \pm 5.14$ & $4.92 \pm 3.44$ & $4.72 \pm 3.46$ & $3.33 \pm 2.50$ \\
1.002 & $6.04 \pm 5.69$ & $5.19 \pm 4.26$ & $4.52 \pm 3.09$ & $3.48 \pm 2.62$ \\
\hline \hline
\end{tabular}

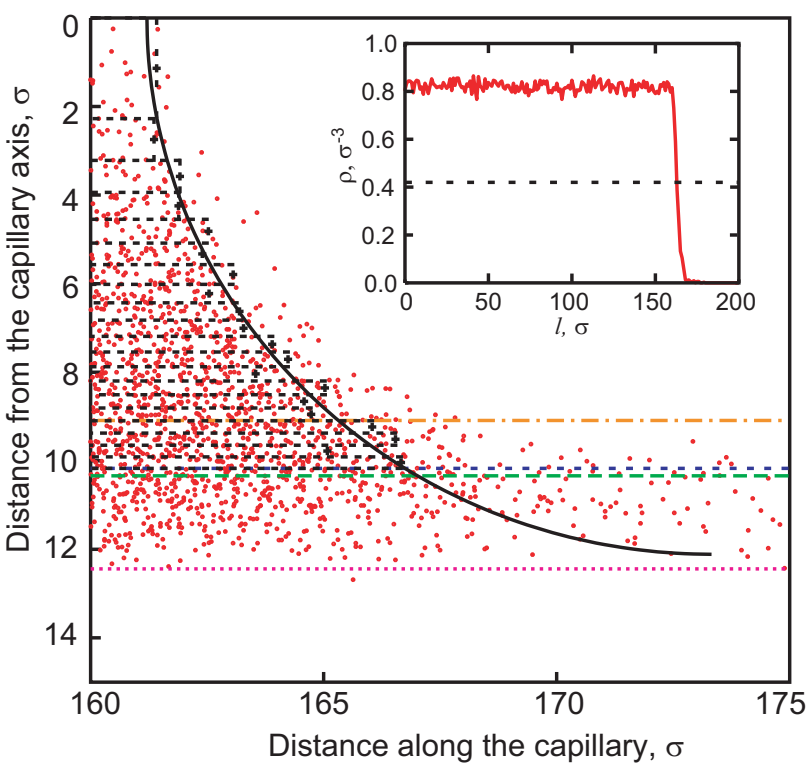

Figure 5: Rising meniscus. Illustration of contact angle calculation. The horizontal lines are indexing $R_{b}=9.09, R_{\theta}=10.17, R_{a v}=10.34$ and $R_{\text {cut }}=12.45$. $(\mathcal{R}=0.805, \varepsilon=1.40$.) The number density along the capillary is shown in the inset.

The position of the meniscus along the capillary $(l(t)$ in equation 3$)$ was set at the intersection of the fitting arc and the capillary axis at time $t$. The fitting circular arc was obtained by minimization of the following residual:

$$
\aleph=\sum_{i=1}^{20}\left(\chi_{i}-\chi\right)^{2}
$$

where $\chi$ is the radius of the arc and $\chi_{i}$ is the distance from the center of the arc to the liquidvapor boundary in the $i$-th bin.

The residuals also provide information about the deviation of the meniscus from a hemispherical shape. Although the error bars are of the same order of magnitude as the values themselves, the observable trend is that the deviation is stronger for rough capillaries and for capillaries of low wettability, where fluctuations are higher (2).

The distance from the central axis used as a reference for the measurement of the contact angle $\left(R_{\theta}\right)$ can be chosen arbitrarily for rough capillaries. For instance $R_{\theta}$ may be set as the distance from the center line to the largest as- 
perity $\left(R_{b}\right)$, or it could be the average roughness distance $\left(R_{a v}\right)$, or the limit distance where the fluid particles start feeling the outer wall of the capillary $\left(R_{\text {cut }}\right)$ (those lines are shown in 5 ).

For capillaries of different roughnesses $R_{b}$ and $R_{a v}$ may be different. To facilitate comparison the contact angles must be measured at the same distance from the capillary axis for all cases. An additional requirement is that the angle has to be measured in the vicinity of the wall, where the roughness has influence; however it cannot be too deep inside the roughness layer where the fluid particles fill out the empty space between the asperities on the capillary wall instead of forming a continuum media. Within those constraints we have chosen $R_{\theta}=10.17 \sigma$. This distance corresponds to the average roughness of the long capillary with the highest roughness $(\mathcal{R}=1.002 \sigma)$.

For the smoothest capillary $(\mathcal{R}=0.145 \sigma)$ we used $R_{\theta}=R_{a v}=11.63 \sigma$ which is noticeably larger than the previous one, simply because the capillary effective radius is larger. This mandatory distinction somewhat limits the validity of the comparisons between this capillary and the rough ones.

\section{Results and Discussion}

As the result of the simulation runs, we obtained the position of the meniscus and the dynamic contact angle as a function of time in capillaries of different roughness and wettability. The instantaneous values of the meniscus position and contact angle were calculated from the system "snapshots" according to the procedure described in the previous section. The snapshots were taken every 100 timesteps for the long capillaries and every 10 timesteps for the short ones.

As the start of the imbibition $(t=0)$ we considered the moment when the meniscus passes the level:

$$
\begin{aligned}
& \left.l\right|_{\text {start }}=0 \sigma, \quad \text { capillary length } 100 \sigma \\
& \left.l\right|_{\text {start }}=5 \sigma, \quad \text { capillary length } 200 \sigma,
\end{aligned}
$$

which corresponds to the positions of the imper-
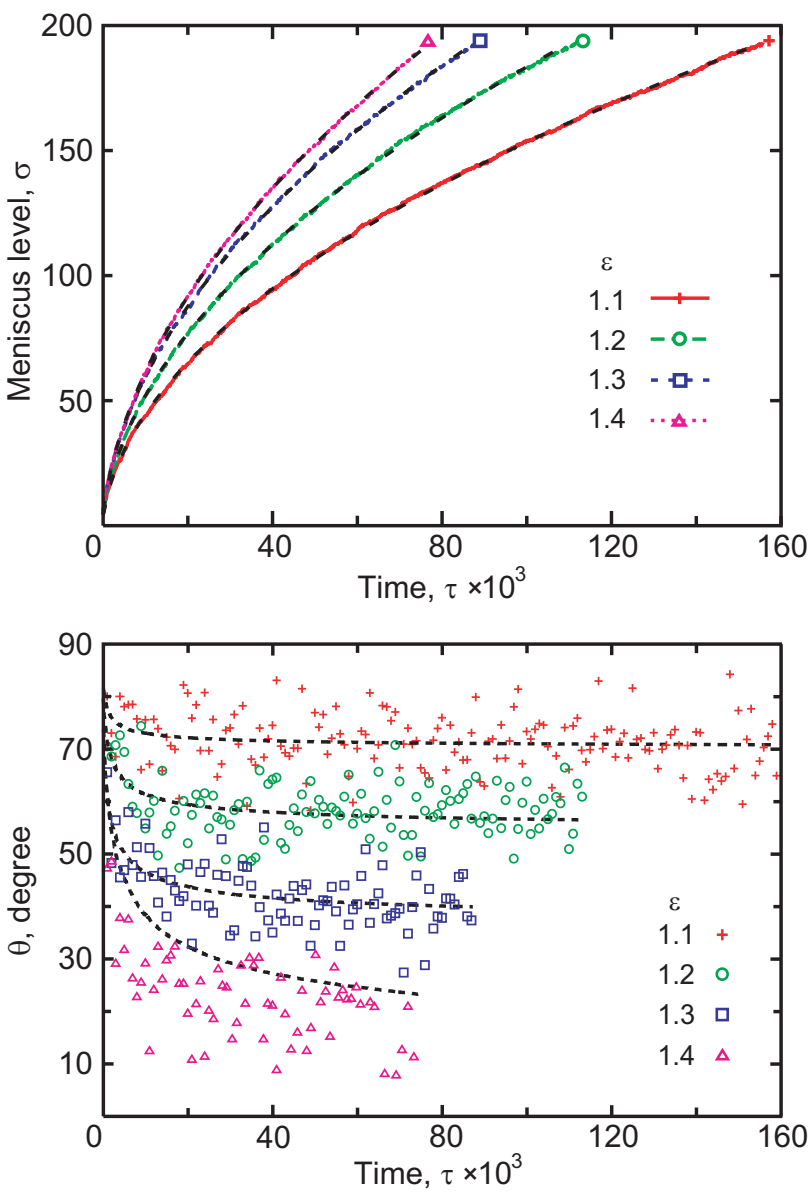

Figure 6: Long capillary $\left(\mathcal{R}=0.145, R_{\theta}=\right.$ 11.63). The dashed lines correspond to the best fit of the meniscus position and the contact angle with 9 and 12, respectively. For the contact angle data the decimation is 1:10.

meable barriers during the equilibration phase.

We have set the limits for the calculation of the position of the rising meniscus as:

$$
\begin{aligned}
& \left.l_{c}\right|_{\text {end }}=98 \sigma, \quad \text { capillary length } 100 \sigma \\
& \left.l_{c}\right|_{\text {end }}=198 \sigma, \quad \text { capillary length } 200 \sigma \text {. }
\end{aligned}
$$

Where $l_{c}$ corresponds to the level where the fitting arc crosses the $R_{\theta}$ line, this ensures that the meniscus is not affected by the capillary end.

\section{Smoothing data by averaging in classes} of roughness: Since the simulations were performed for three sets of capillaries (long, short, short-shifted) the data were averaged for close values of roughness $\mathcal{R}$ (i.e. 0.381 , $0.375,0.348)$ and same value of wettability $\varepsilon$. 


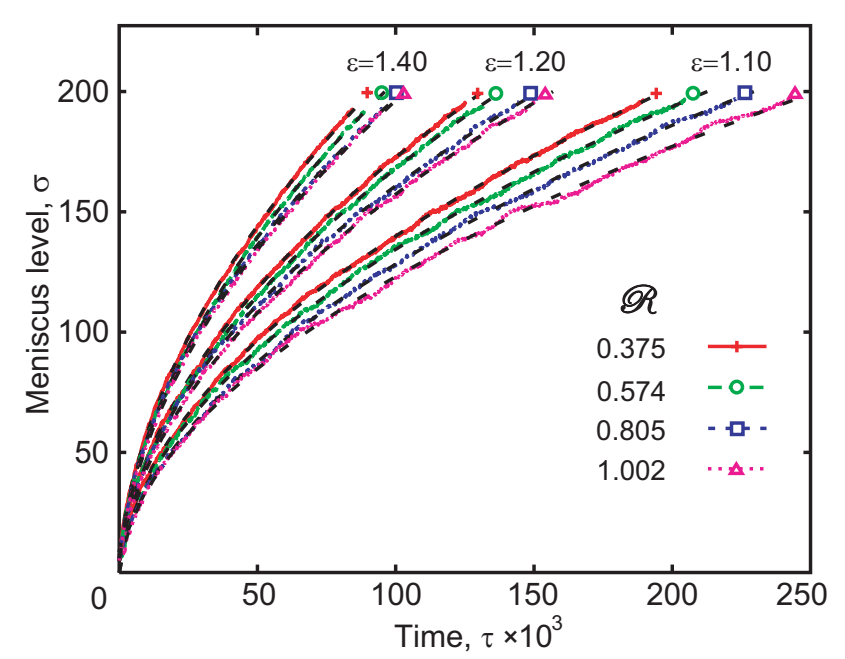

Figure 7: Long capillary $(\varepsilon=1.10,1.20,1.40$, $\mathcal{R}=0.375,0.574,0.805,1.002)$. The dashed lines correspond to the best fit with 9 .

Thus the value of any parameter $A$ was averaged as $\langle A\rangle=\frac{1}{N_{c a p}} \sum_{i}^{N_{c a p}} A_{i}, N_{c a p}$ corresponds to the number of the capillaries used for the averaging and was equal to 1, 2 or 3 depending on the particular $\varepsilon$ and the roughness group ( 3 for $\varepsilon=1.10,1.20,1.30,1.40,2$ for $\varepsilon=1.15,1.25,1.35,1$ for the smoothest capillary $\mathcal{R}=0.145)$. This averaged value $\langle A\rangle$ was then subscribed to one of the five classes of roughnesses $\langle\mathcal{R}\rangle=\frac{1}{N_{\text {cap }}} \sum_{i}^{N_{\text {cap }}} \mathcal{R}_{i}, N_{\text {cap }}=$ 3 for $\mathcal{R} \neq 0.145 ; N_{\text {cap }}=1$ for $\mathcal{R}=0.145$.

\section{Imbibition curves and dynamic contact} angle: The imbibition curves were fitted with the modified Lucas-Washburn equation (9) and the contact angle dependences were fitted with 12. For the long capillaries the simulation data are plotted along with the corresponding approximation curves in 6,7 and 8 (the plots for the short capillaries are not displayed). The modified Lucas-Washburn equation (9) perfectly fits the curves. As it can be seen in 9 the classical Lucas-Washburn equation is not able to properly describe the imbibition dynamics at the initial stage of the process. Additional information about the quality of the fit is provided in the inset in 9, where the difference $\Delta l$ between simulation data and the fitting curves is displayed as a function of the penetra-

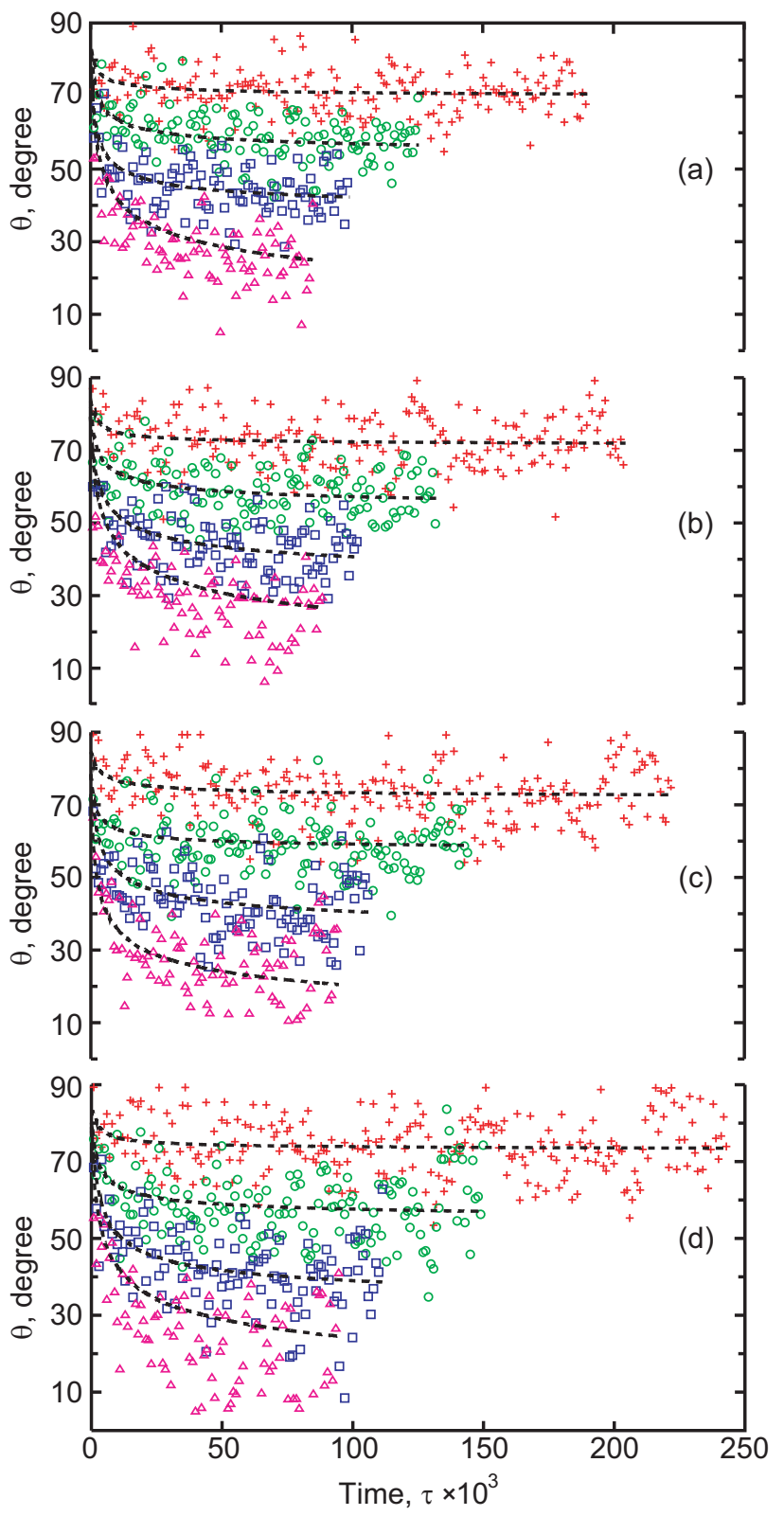

Figure 8: Dynamic contact angle as a function of time for long capillaries of different roughness: $\mathcal{R}=0.375(a), 0.574(\mathrm{~b}), 0.805(\mathrm{c})$, $1.002(\mathrm{~d})$; and wettability: $\varepsilon=1.10(+)$, $1.20(\bigcirc), 1.30(\square), 1.40(\triangle)$. The dashed lines correspond to the best fit of contact angle with 12. The data decimation is $1: 10$.

tion length. Our data support the conclusions of Martic et al. ${ }^{6}$ that this difference is getting stronger when the interaction between liquid and solid increases and that the penetration distance where the dynamic contact angle should be taken into account is of order of $R \xi / \eta$, which for our system is in the range of $4 R-9 R$. 


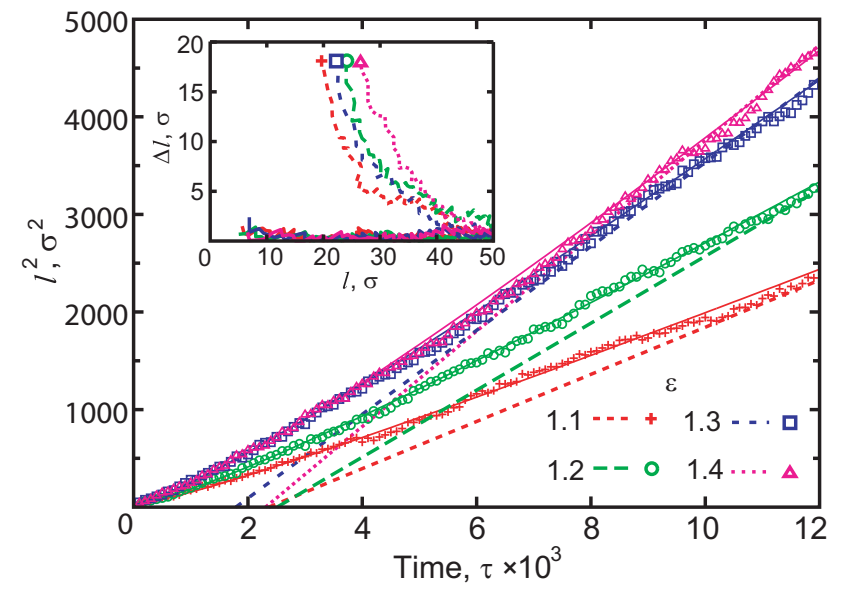

Figure 9: Squared imbibition length as a function of time. Simulation data (symbols) with corresponding best fits: classical LucasWashburn equation (3) (dashed lines) and modified Lucas-Washburn equation (9) (thin solid lines). Difference $\Delta l$ between simulation data and approximation fit as a function of penetration length is shown in the inset. Data for smooth $(\mathcal{R}=0.145)$ long capillaries.

The calculated values of $P_{2}$ and $P_{3}$ obtained by fitting the imbibition curves were used for approximating the dynamic contact angle data with 12. Although the graphs for the dynamic contact angle are very noisy (6 and 8 ) reasonable fittings can be done using 12 . Clearly the approximation of the wettability contact angle is more accurate when the advancing contact angle does not vary significantly during the imbibition (i.e. the approximation is better for smaller $\varepsilon)$. At the same time, the simulation results for high $\varepsilon$ can be used for estimating the scaling dependence of the contact angle $\theta$ versus time. Within the molecular-kinetic regime ${ }^{31}$ this dependence is shown to be $\propto t^{-1 / 4}$ which provides a reasonable approximation of the simulation data (see 10).

The averaged values of $P_{2}, P_{3}$ and $\cos \theta_{e}$ are shown in 3 . (Individual values of the parameters for all the capillaries considered can be found in the supplementary materials. Since the initial position of the rising meniscus was different in simulations with short and long capillaries, average values of $P_{1}$ have no meaning and are not included in 3.) Curves in 7 and

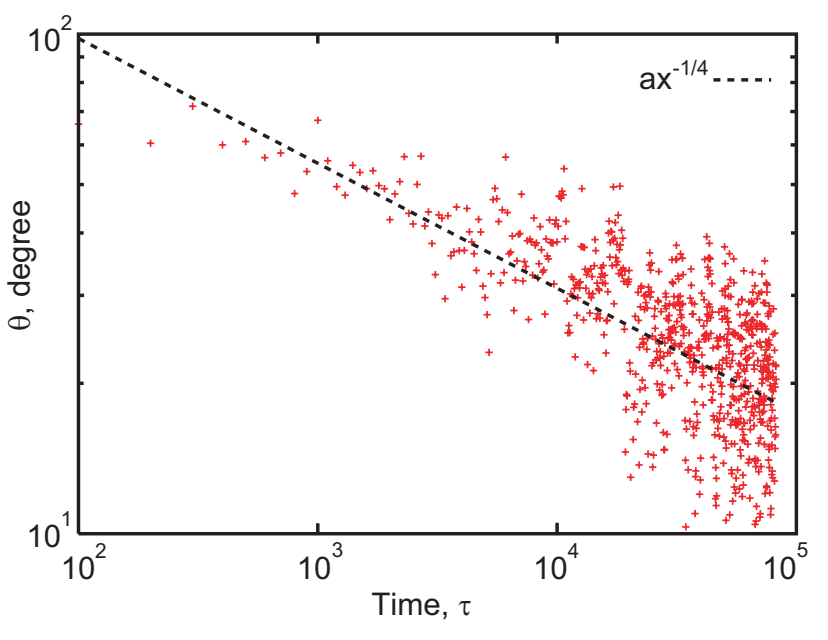

Figure 10: Dynamic contact angle as a function of time $(\mathcal{R}=0.378, \varepsilon=1.40)$.

data in 3 point out that the roughness slows down the imbibition (as expected higher wettability increases the meniscus velocity, also visible in 6). This complies very well with the observation that microroughness inhibits the wetting. ${ }^{79,80}$ For $t \rightarrow \infty$ the meniscus velocity is governed by $P_{3}(10)$. The smaller the $P_{3}-$ the faster the rising. Since $P_{3}=4 \eta /\left(R \gamma \cos \theta_{e}\right)$ where $R=R_{D}^{2} / R_{V}$ and the static radius is slightly larger for smooth capillaries, a side effect on the capillary imbibition may occur (not directly related to the wall roughness). To separate near-to-the-wall effects related to the roughness from the effects related to the capillary radius we consider $R_{D}$ and $R_{V}$ separately.

Volumetric radius $R_{V}$ : The average volumetric radius, already defined as the "cross section" radius $\sqrt{S / \pi}$ in 2 , can be estimated from the bulk number density $\rho_{\text {bulk }}$ and the number of dodecane particles $N_{p}$ in the capillary of length $L_{0}$ :

$$
R_{V}=\sqrt{\frac{N_{p} / \rho_{b u l k}}{\pi L_{0}}},
$$

where $\rho_{\text {bulk }}=0.842$. This approach was used for example by Morrow. ${ }^{2}$

$R_{V}$ was calculated using the middle section $[20 \sigma: 70 \sigma]$ for short capillaries and $[20 \sigma: 170 \sigma]$ for long ones (averaged over 100 different config- 
Table 3: Average values of $P_{2}, P_{3}$ and $\cos \theta_{e}$ obtained by fitting the imbibition curves. Full data set is available in the supplementary materials.

\begin{tabular}{|c|c|c|c|c|}
\hline$\langle\mathcal{R}\rangle$ & $\varepsilon$ & $P_{2}$ & $P_{3}$ & $\begin{array}{r}\cos \theta_{e} \\
\pm 0.003\end{array}$ \\
\hline \multicolumn{5}{|c|}{$R_{\theta}=11.63$} \\
\hline 0.145 & 1.40 & $62.4 \pm 2.6$ & $3.47 \pm 0.08$ & 0.998 \\
\hline 0.145 & 1.35 & $45.5 \pm 0.4$ & $3.92 \pm 0.00$ & 0.926 \\
\hline 0.145 & 1.30 & $44.0 \pm 0.5$ & $4.26 \pm 0.01$ & 0.808 \\
\hline 0.145 & 1.20 & $60.1 \pm 0.7$ & $5.30 \pm 0.01$ & 0.584 \\
\hline 0.145 & 1.10 & $57.1 \pm 0.7$ & $7.78 \pm 0.01$ & 0.341 \\
\hline \multicolumn{5}{|c|}{$R_{\theta}=10.17$} \\
\hline 0.368 & 1.40 & $65.5 \pm 8.4$ & $3.93 \pm 0.16$ & 0.965 \\
\hline 0.368 & 1.35 & $45.4 \pm 5.1$ & $4.69 \pm 0.13$ & 0.845 \\
\hline 0.368 & 1.30 & $63.5 \pm 12.9$ & $4.58 \pm 0.08$ & 0.804 \\
\hline 0.368 & 1.25 & $59.2 \pm 0.4$ & $5.05 \pm 0.11$ & 0.687 \\
\hline 0.368 & 1.20 & $74.7 \pm 13.8$ & $5.86 \pm 0.38$ & 0.582 \\
\hline 0.368 & 1.15 & $77.8 \pm 26.0$ & $7.00 \pm 0.35$ & 0.462 \\
\hline 0.368 & 1.10 & $83.4 \pm 5.4$ & $9.11 \pm 0.38$ & 0.345 \\
\hline 0.565 & 1.40 & $65.6 \pm 18.0$ & $4.22 \pm 0.21$ & 0.956 \\
\hline 0.565 & 1.35 & $62.6 \pm 5.7$ & $4.52 \pm 0.01$ & 0.890 \\
\hline 0.565 & 1.30 & $82.6 \pm 5.3$ & $4.60 \pm 0.02$ & 0.838 \\
\hline 0.565 & 1.25 & $59.3 \pm 7.2$ & $5.86 \pm 0.16$ & 0.666 \\
\hline 0.565 & 1.20 & $79.4 \pm 16.1$ & $6.40 \pm 0.32$ & 0.565 \\
\hline 0.565 & 1.15 & $70.5 \pm 9.3$ & $7.75 \pm 0.05$ & 0.428 \\
\hline 0.565 & 1.10 & $80.1 \pm 20.9$ & $10.04 \pm 0.22$ & 0.318 \\
\hline 0.794 & 1.40 & $65.0 \pm 6.2$ & $4.47 \pm 0.13$ & 0.967 \\
\hline 0.794 & 1.35 & $61.8 \pm \quad 4.9$ & $4.73 \pm 0.07$ & 0.897 \\
\hline 0.794 & 1.30 & $69.3 \pm 11.6$ & $5.09 \pm 0.21$ & 0.796 \\
\hline 0.794 & 1.25 & $67.3 \pm \quad 7.9$ & $5.88 \pm 0.29$ & 0.672 \\
\hline 0.794 & 1.20 & $76.6 \pm 18.5$ & $6.69 \pm 0.29$ & 0.558 \\
\hline 0.794 & 1.15 & $72.9 \pm 2.7$ & $8.43 \pm 0.10$ & 0.408 \\
\hline 0.794 & 1.10 & $119.8 \pm 37.7$ & $10.29 \pm 0.67$ & 0.309 \\
\hline 0.997 & 1.40 & $74.5 \pm 14.0$ & $4.55 \pm 0.31$ & 0.977 \\
\hline 0.997 & 1.35 & $70.6 \pm 1.1$ & $4.99 \pm 0.14$ & 0.917 \\
\hline 0.997 & 1.30 & $78.9 \pm 14.0$ & $5.15 \pm 0.30$ & 0.839 \\
\hline 0.997 & 1.25 & $74.7 \pm 14.4$ & $6.05 \pm 0.19$ & 0.693 \\
\hline 0.997 & 1.20 & $90.1 \pm 21.7$ & $7.04 \pm 0.51$ & 0.583 \\
\hline 0.997 & 1.15 & $102.2 \pm 16.4$ & $8.30 \pm 0.74$ & 0.450 \\
\hline 0.997 & 1.10 & $133.0 \pm 74.7$ & $10.95 \pm 1.62$ & 0.328 \\
\hline
\end{tabular}

urations when the section was entirely filled by fluid). The calculated $R_{V}$ (4) increases as the roughness decreases. Although we have found that for capillaries of the same roughness, $R_{V}$ is slightly larger for higher $\varepsilon$, the difference remaining smaller than $1 \%$. The slight increase of the particle number in the capillary when increasing the wettability is due to a better packing and a decrease in the mobility of the particles close to the capillary wall.

Variation of $P_{3} \cos \theta_{e}$ : For the capillaries belonging to the same class of roughness the product $P_{3} \cos \theta_{e}$ should be constant (8); however $P_{3} \cos \theta_{e}$ increases with the wettability parameter (see 11). This observation is also supported by the data of Martic et al. ${ }^{31}$ though they did not focus on this discrepancy.
Table 4: Volumetric radii of the capillaries, $R_{V}$ (precision 0.01).

\begin{tabular}{c|ccccc}
\hline \hline & & \multicolumn{5}{|c}{$\langle\mathcal{R}\rangle$} \\
$\varepsilon$ & 0.997 & 0.794 & 0.565 & 0.368 & 0.145 \\
\hline 1.10 & 10.82 & 10.84 & 10.90 & 11.07 & 11.56 \\
1.15 & 10.83 & 10.85 & 10.92 & 11.08 & \\
1.20 & 10.86 & 10.87 & 10.94 & 11.10 & 11.56 \\
1.25 & 10.87 & 10.89 & 10.95 & 11.11 & \\
1.30 & 10.89 & 10.91 & 10.97 & 11.13 & 11.61 \\
1.35 & 10.89 & 10.92 & 10.98 & 11.14 & 11.60 \\
1.40 & 10.92 & 10.93 & 10.99 & 11.15 & 11.62 \\
\hline \hline
\end{tabular}

The only reason for the product $P_{3} \cos \theta_{e}$ to be not a constant is that between the parameters $R, \eta$ and $\gamma$, some or all vary. The surface tension $\gamma$ represents the fluid properties at the liquid-vapor interface and should be independent on $\varepsilon$. The interaction with the wall can result in a local increase of the fluid viscosity; however viscosity in the central part of the capillary should not be affected. This effect can be described by a slip over a "sticky surface layer" 81 and will affect the hydrodynamic radius of the capillary. $1 \%$ of variation of $R_{V}$ (see 4 ) can not completely explain the deviation described above. The only remaining parameter which can significantly change its value due to wettability is the hydrodynamical radius $R_{D}$. It can be calculated as

$$
R_{D}=\sqrt{\frac{4 \eta R_{V}}{P_{3} \gamma \cos \theta_{e}}} .
$$

Difference $\left(R_{D}-R_{V}\right)$ as a function of wettability $\varepsilon$, and roughness $\mathcal{R}$ : In the proximity of the wall, the effects of roughness and wettability on the flow are conveniently described by the difference $\Delta R=R_{D}-R_{V}$. In 12 $\Delta R$ is shown as a function of $1 / \varepsilon^{2}$. The choice is based on the evidence ${ }^{82,83}$ that the slip length $\delta$ depends on the wettability as $\delta \propto 1 / \varepsilon^{2}$, and we expect the similar dependence for $\Delta R$. In addition the dependence of $\Delta R$ on the capillary roughness (13) shows a linear decay ${ }^{84}$ suggesting that the difference $\Delta R$ can be expressed as

$$
\Delta R=\Delta R_{0}+\frac{k_{\varepsilon}}{\varepsilon^{2}}+k_{\mathcal{R}} \mathcal{R} .
$$




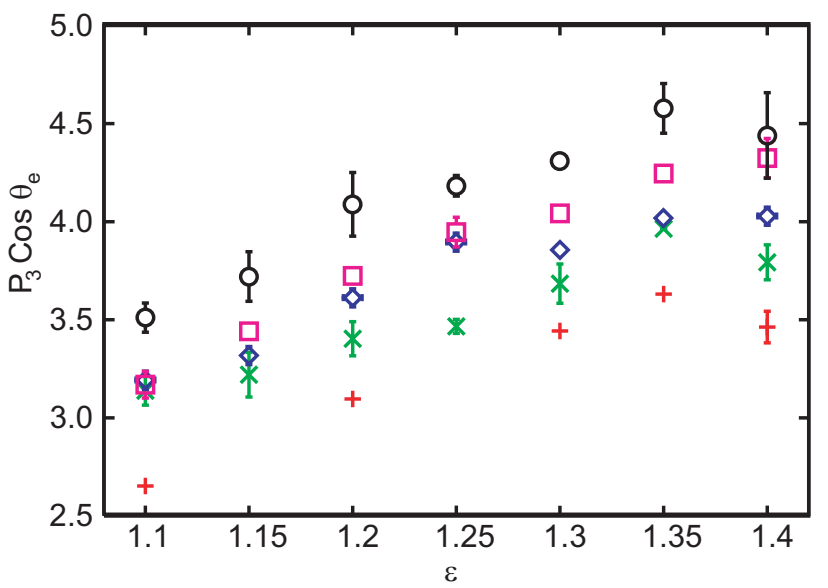

Figure 11: Product $P_{3} \cos \theta_{e}$ as a function of $\varepsilon$ for capillaries of different classes of roughness: $\langle\mathcal{R}\rangle=0.145(+), 0.368(\times), 0.565(\diamond), 0.794(\square)$, $0.997(\bigcirc)$.

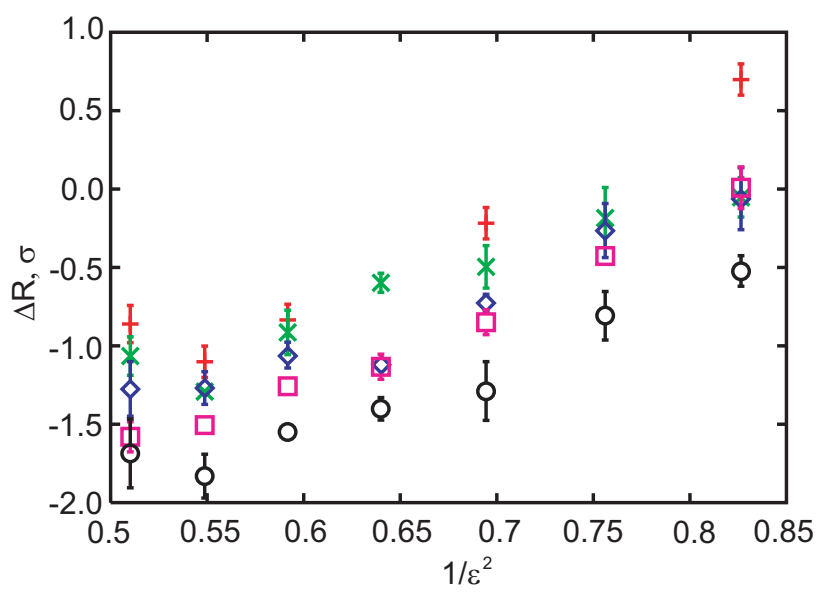

Figure 12: $\Delta R$ as a function of $1 / \varepsilon^{2}$ for capillaries of different classes of roughness: $\langle\mathcal{R}\rangle=0.145(+), 0.368(\times), 0.565(\diamond), 0.794(\square)$, $0.997(\bigcirc)$.

Here $\Delta R_{0}$ is the asymptotic value of $\Delta R$ for $\varepsilon \rightarrow \infty$ and $\mathcal{R} \rightarrow 0$ and $k_{\varepsilon}, k_{\mathcal{R}}$ are numerical coefficients. $k_{\varepsilon}=5.07 \pm 1.01$ was obtained by fitting the data in 12 , and $k_{\mathcal{R}}=-1.00 \pm 0.18$ by fitting the data in 13. From the data it is clearly visible that the case $\varepsilon=1.40\left(\frac{1}{\varepsilon^{2}} \approx 0.51\right)$ systematically deviates from the main patterns (during the fitting procedure the corresponding points were omitted). This case corresponds to an extreme wetting situation which requires additional investigations.

The values of $\Delta R_{0}$ calculated from the simu-

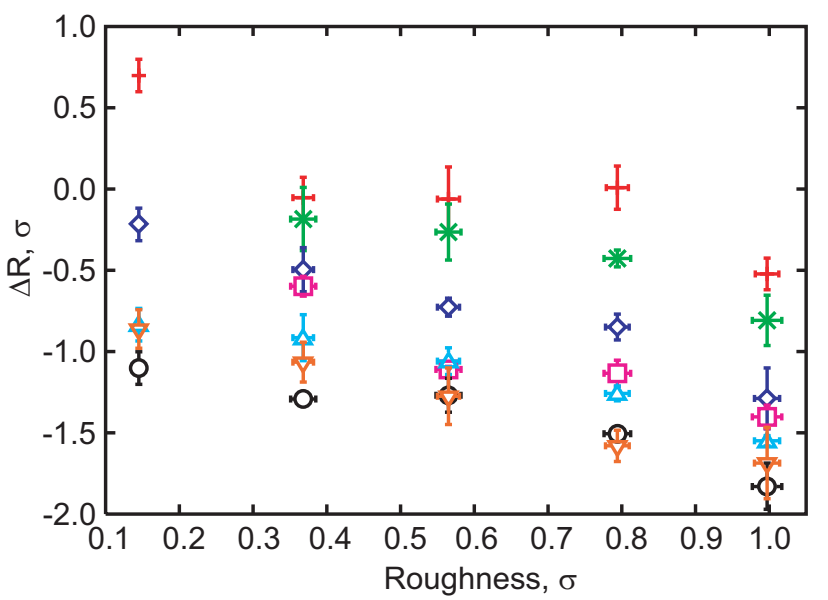

Figure 13: $\Delta R$ as a function of roughness for capillaries of different wettability: $\varepsilon=1.10(+)$, $1.15(\times), 1.20(\diamond), 1.25(\square), 1.30(\triangle), 1.35(\nabla)$, $1.40(\bigcirc)$.

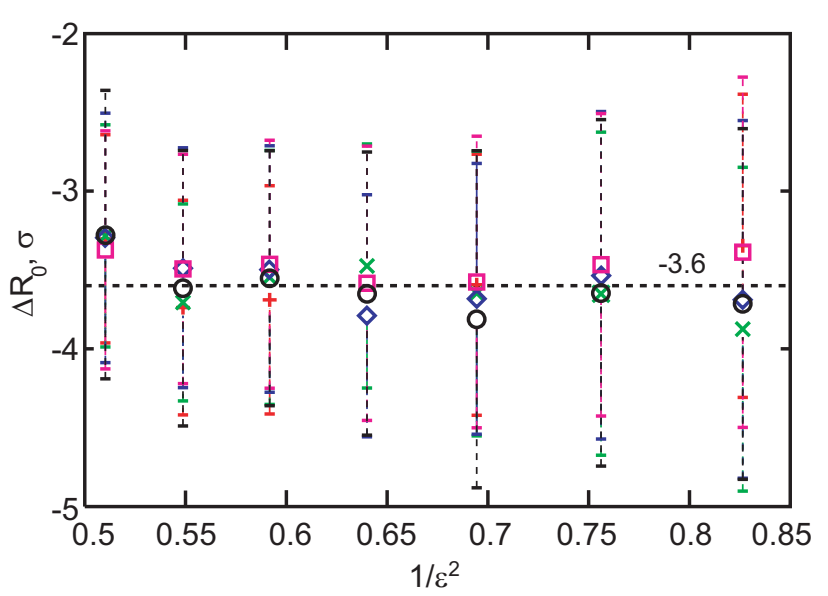

Figure 14: $\Delta R_{0}$ as a function of $1 / \varepsilon^{2}$ for capillaries of different classes od roughness: $\langle\mathcal{R}\rangle=0.145(+), 0.368(\times), 0.565(\diamond), 0.794(\square)$, $0.997(\bigcirc)$.

lation data for different $\mathcal{R}$ and $\varepsilon$ are presented in 14. All the data are distributed around a single line $\Delta R_{0}=-3.60$, which proves the validity of 24. The layer thickness of immobilized fluid one expects to have in case of $\varepsilon \rightarrow \infty$ is equal to the interaction cut-off radius $r_{c}, \Delta R_{0}$ well corresponds to $r_{c}$ plus one $\sigma$ of stand off showing the consistency of our fitting approach.

Wetting line friction coefficient: The coefficient of wetting line friction introduced in 6 can be calculated from $P_{2}$ as $\xi=P_{2} \gamma \cos \theta_{e}$. 
Unfortunately, the typical $P_{2}$ error bars are quite large limiting the possibility of accurate data analysis. Nevertheless, within the frame of this uncertainty some estimates can be performed. We calculated $\xi$ from $P_{2}$ (provided in 3) and using equation (7) from Ref. 62 based on molecular-kinetics theory ${ }^{61,62}$ approach:

$$
\xi=\frac{\eta}{\rho_{b u l k} \lambda^{3}} \exp \left(\frac{\gamma\left(1+\cos \theta_{e}\right) \lambda^{2}}{k_{B} T}\right) .
$$

The average length of molecular displacement $\lambda$ is estimated according to de Ruijter et al. ${ }^{85}$ as the distance between the centers of the first and the second layer near to the capillary wall. In our case $\lambda$ is about $0.95 \sigma$ (see 4 ). The results are rather dispersed, however the simulation data and the values obtained from 25 show similar trend and are in quantitative agreement (see 15). In both cases $\xi$ clearly increases with the increase of the wettability. Intuitively one also expects $\xi$ to increase with roughness resulting in a regular and ordered vertical spread of data for each value of wettability, which is suggested but not firmly demonstrated due to the poor data precision on $P_{2}$. The comparison of the simulation and the theory looks very promising and can be consider as an additional support for the validity of the molecular-kinetic theory.

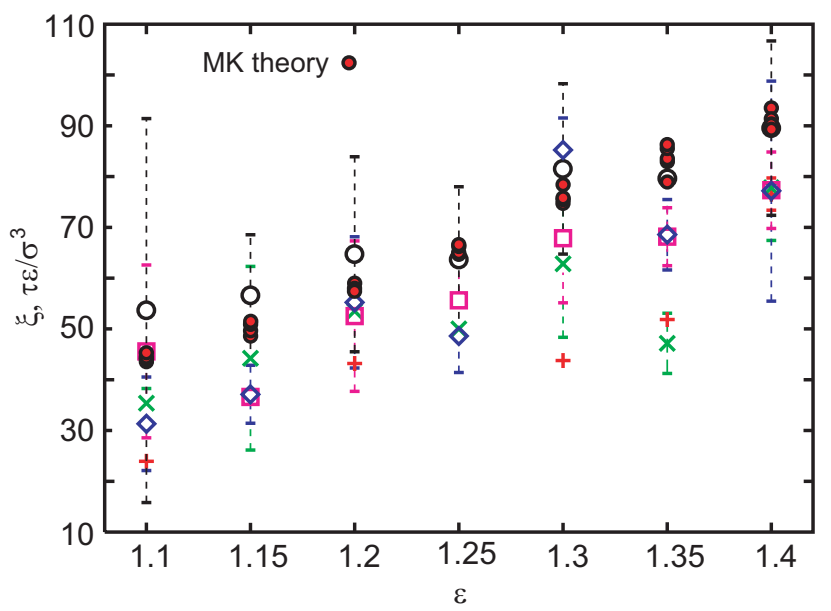

Figure 15: Coefficient of the wetting line friction $\xi$ as a function of $\varepsilon$ for capillaries of different classes of roughness: $\langle\mathcal{R}\rangle=0.145(+)$,

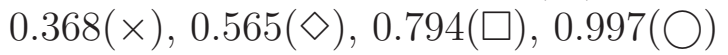

\section{Conclusions}

In this study an extensive investigation of the effect of capillary wettability and roughness on the imbibition dynamics was carried out by Molecular Dynamics simulation. The capillary rise of a fluid can be perfectly represented by the Lucas-Washburn equation modified to take into account the dynamic contact angle, which was described using the molecular kinetics theory.

The roughness is shown to slow down the meniscus velocity. The increase of the roughness and/or the capillary wettability induces an effective decrease of the hydrodynamics radius of the capillary resulting in a linear relation between $\Delta R, \mathcal{R}$ and $1 / \varepsilon^{2}$. The study also demonstrates the relevance of molecular dynamics for the description of the triple line motion on surfaces of random roughnesses.

\section{Acknowledgement}

The authors are grateful to J. R. A. Pearson, P. S. Hammond, S. Dyer, A. Clarke, E. Lac and B. Nicot for their comments and stimulating discussions.

Supporting information Values of the fitting parameters for each modeling case are provided as supporting information. This information is available free of charge via the Internet at http://pubs.acs.org/.

\section{References}

(1) Wenzel, R. N. Ind. Eng. Chem. 1936, 28, 988-994.

(2) Morrow, N. R. J. Can. Petr. Tech. 1975, $14,42-53$.

(3) Quéré, D. Annu. Rev. Mater. Res. 2008, 38, 71-99.

(4) Morrow, N. R. J. Can. Petr. Tech. 1976, 15, 49-69.

(5) Anderson, W. G. J. Pet. Technol. 1987, 39, 1283-1300. 
(6) Martic, G.; De Coninck, G.; Blake, T. D. J. Colloid Interface Sci. 2003, 263, 213216.

(7) De Gennes, P. G. Rev. Mod. Phys. 1985, 57, 827-863.

(8) Dussan V., E. B. Ann. Rev. Fluid Mech. 1979, 11, 371-400.

(9) Ralston, J.; Popescu, M.; Sedev, R. Annu. Rev. Mater. Res. 2008, 38, 23-43.

(10) Stange, M.; Dreyer, M. E.; Rath, H. J. Phys. Fluids 2003, 15, 2587-2601.

(11) Fries, N.; Dreyer, M. J. Colloid Interface Sci. 2009, 338, 514-518.

(12) Hilpert, M. J. Colloid Interface Sci. 2009, $33 \%, 138-144$.

(13) Hilpert, M. J. Colloid Interface Sci. 2009, 337, 131-137.

(14) Chen, C.; Gao, C.; Zhuang, L.; Li, X.; Wu, P.; Dong, J.; Lu, J. Langmuir 2010, 26, 9533ढ̈9538.

(15) Blake, T. D. J. Colloid Interface Sci. 2006, 299, 1-13.

(16) Siebold, A.; Nardin, M.; Schultz, J.; Walliser, A.; Oppliger, M. Colloids Surf. A 2000, 161, 81-87.

(17) van Delft, K. M.; Eijkel, J. C.; Mijatovic, D.; Druzhinina, T. S.; Rathgen, H.; Tas, N. R.; Berg, A. v. d.; Mugele, F. Nano Lett. 2007, \%, 345-350.

(18) Tas, N. R.; Haneveld, J.; Jansen, H. V.; Elwenspoek, M.; van den Berg, A. Appl. Phys. Lett. 2004, 85, 3274-3276.

(19) Han, A.; Mondin, G.; Hegelbach, N. G.; Rooij, N. F. d.; Staufer, U. J. Colloid Interface Sci. 2006, 293, 151-157.

(20) Huber, P.; Grüer, S.; Schäfer, C.; Knorr, K.; Kityk, A. V. Eur. Phys. J. ST 2007, 141, 101-105.
(21) Gruener, S.; Huber, P. Phys. Rev. Lett. 2009, 103, 174501.

(22) Gruener, S.; Hoffman, T.; Wallacher, D.; Kityk, A. V.; Huber, P. Phys. Rev. E 2009, 79, 067301.

(23) Gogotsi, Y.; Libera, J. A.; GüvencYazicioglu, A.; Megaridis, C. M. Appl. Phys. Lett. 2001, 79, 1021-1023.

(24) Gelb, L. D.; Hopkins, A. C. Nano Lett. 2002, 2, 1281-1285.

(25) Henrich, B.; Cupelli, C.; Santer, M.; Moseler, M. New J. Phys. 2008, 10, 113022 .

(26) Supple, S.; Quirke, N. Phys. Rev. Lett 2003, 90, 214501.

(27) Koplik, J.; Banavar, J. R. Annu. Rev. Fluid Mech. 1995, 27, 257-292.

(28) Blake, T. D.; Clarke, A.; De Coninck, J.; de Ruijter, M. J. Langmuir 1997, 13, 2164-2166.

(29) De Coninck, J.; Blake, T. D. Ann. Rev. Mater. Res. 2008, 38, 1-22.

(30) Heine, D. R.; Grest, G. S.; Webb III, E. B. Phys. Rev. E 2004, 70, 011606.

(31) Martic, G.; Gentner, F.; Seveno, D.; De Coninck, J.; Blake, T. D. J. Colloid Interface Sci. 2004, 270, 171-179.

(32) Martic, G.; Gentner, F.; Seveno, D.; Coulon, D.; De Coninck, J.; Blake, T. D. Langmuir 2002, 18, 7971-7976.

(33) Supple, S.; Quirke, N. J. Chem. Phys 2004, 121, 8571-8579.

(34) Supple, S.; Quirke, N. J. Chem. Phys. 2005, 122, 104706.

(35) Thompson, P. A.; Robbins, M. O. Phys. Rev. Lett. 1989, 63, 766-769.

(36) Dimitrov, D. I.; Milchev, A.; Binder, K. Phys. Rev. Lett. 2007, 99, 054501. 
(37) Martic, G.; Blake, T. D.; De Coninck, J. Langmuir 2005, 21, 11201-11207.

(38) Jin, W.; Koplik, J.; Banavar, J. R. Phys. Rev. Lett. 1997, 78, 1520-1523.

(39) Ahadian, S.; Kawazoe, Y. Colloid Polym. Sci. 2009, 287, 961-967.

(40) Webb III, E. B.; Hoyt, J. J. Acta Materialia 2008, 56, 1802-1812.

(41) Chin, J.; Boek, E. S.; Coveney, P. V. Phil. Trans. R. Soc. Lond. A 2002, 360, 547558.

(42) Diotallevi, F.; Biferale, L.; Chibbaro, S.; Lamura, A.; Pontrelli, G.; Sbragaglia, M.; Succi, S.; Toschi, F. Eur. Phys. J. ST 2009, 166, 111-116.

(43) Diotallevi, F.; Biferale, L.; Chibbaro, S.; Pontrelli, G.; Toschi, F.; Succi, S. Eur. Phys. J. ST 2009, 171, 237-243.

(44) Chibbaro, S.; Biferale, L.; Diotallevi, F.; Succi, S. Eur. Phys. J. ST 2009, 171, 223-228.

(45) Wolf, F. G.; dos Santos, L. O. E.; Philippi, P. C. J. Colloid Interface Sci. 2010, 344, 171-179.

(46) Galea, T. M.; Attard, P. Langmuir 2004, 20, 3477-3482.

(47) Niavarani, A.; Priezjev, N. V. Phys. Fluids 2009, 21, 052105.

(48) Liou, W. W.; Peng, Y.; Parker, P. E. J. Colloid Interface Sci. 2009, 333, 389-399.

(49) Lucas, R. Kolloid Z 1918, 23, 15.

(50) Washburn, E. W. Phys. Rev. 1921, 17, 273.

(51) Bosanquet, C. H. Philos. Mag. 1923, 45, 525.

(52) Clarke, A.; Stattersfield, E. Phys. Fluids 2006, 18, 048106.

(53) Bayer, I. S.; Megaridis, C. M. J. Fluid. Mech. 2006, 558, 415-449.
(54) Hoffman, R. L. J. Colloid Interface Sci. 1975, 50, 228.

(55) Popescu, M. N.; Ralston, J.; Sedev, R. Langmuir 2008, 24, 12710-12716.

(56) Tanner, L. H. J. Phys. D: Appl. Phys. 1979, 12, 1473-1484.

(57) Joos, P.; Van Remoortere, P.; Bracke, M. J. Colloid Interface Sci. 1989, 136, 189197.

(58) Shikhmurzaev, Y. D. Int. J. Multiphase Flow 1993, 19, 589-610.

(59) Cox, R. G. J. Fluid. Mech. 1998, 35\%, 249-278.

(60) Kalliadasis, S.; Chang, H.-C. Phys. Fluids 1994, 6, 12-23.

(61) Blake, T. D.; Haynes, J. M. J. Colloid Interface Sci. 1969, 30, 421-423.

(62) Bertrand, E.; Blake, T. D.; De Coninck, J. J. Phys.: Condens. Matter 2009, 21, 464124.

(63) Marrink, S. J.; de Vries, A. H.; Mark, A. E. J. Phys. Chem. B 2004, 108, 750-760.

(64) Marrink, S. J.; Risselada, H. J.; Yefimov, S.; Tieleman, D. P.; de Vries, A. H. J. Phys. Chem. B 2007, 111, 7812-7824.

(65) Limbach, H.-J.; Arnold, A.; Mann, B. A.; Holm, C. Comput. Phys. Commun. 2006, $174,704-727$.

(66) Hoogerbrugge, P. J.; Koelman, J. M. V. A. Europhys. Lett. 1992, 19, 155-160.

(67) Epanol, P.; Warren, P. B. Europhys. Lett. 1995, 30, 191-196.

(68) Soddemann, T.; Dünweg, B.; Kremer, K. Phys. Rev. E 2003, 68, 046702.

(69) Allen, M. P.; Tildesley, D. J. Computer Simulation of Liquids; Clarendon, Oxford, 1990. 
(70) Landau, L. D.; Lifshitz, E. M. Statistical Physics Part 1(3rd Edition); Pergamon Press, 1986.

(71) von Meerwall, E.; Beckman, S.; Jang, J.; Mattice, W. L. J. Chem. Phys. 1998, 108, 4299-4304.

(72) Cumming, P. T.; Evans, D. J. Ind. Eng. Chem. Res. 1992, 31, 1237-1252.

(73) Sumner, A. L.; Menke, E. J.; Dubowski, Y.; Newberg, J. T.; Penner, R. M.; Hemminger, J. C.; Wingen, L. M.; Brauersc, T.; FinlaysonPitts, B. J. Phys. Chem. Chem. Phys. 2004, 6, 604-613.

(74) Tang, S. K.; Vassiliev, V. Y.; Mridha, S.; Chan, L. H. Thin Solid Films 1999, 352, 77-84.

(75) Cupelli, C.; Henrich, B.; Glatzel, T.; Zengerle, R.; Moseler, M.; Santer, M. New J. Phys. 2008, 10, 043009.

(76) Caupin, F.; Cole, M. W.; Balibar, S.; Treiner, J. Europhys. Lett. 2008, 82, 56004 .

(77) Quéré, D. Europhys. Lett. 1997, 39, 533538.

(78) Saam, W. F.; Cole, M. W. Phys. Rev. B 1975, 11, 1086-1105.

(79) Semal, S.; Blake, T. D.; Geskin, V.; de Ruijter, M. J.; Castelein, G.; De Coninck, J. Langmuir 1999, 15, 8765-8770.

(80) Savva, N.; Kalliadasis, S.; Pavliotis, G. A. Phys. Rev. Lett. 2010, 104, 084501.

(81) Servantie, J.; Müller, M. Phys. Rev. Lett. 2008, 101, 026101.

(82) Müller, M.; Pastorino, C.; Servantie, J. Comput. Phys. Commun. 2009, 180, 600604.

(83) Bocquet, L.; Barrat, J.-L. Phys. Rev. E 1994, 49, 3079-3092.
(84) Yang, S. C.; Fang, L. B. Mol. Sim. 2005, 31, 971-977.

(85) de Ruijter, M. J.; Blake, T. D.; De Coninck, J. Langmuir 1999, 15, 7836-7847. 